\title{
1,2-Addition or Enolization? Variable Reactivity of a Cerium Acetylide Complex toward Carbonyl Compounds
}

\author{
Jee Eon Kim, Alexander V. Zabula, Patrick J. Carroll, and Eric J. Schelter*
}

P. Roy and Diana T. Vagelos Laboratories, Department of Chemistry, University of Pennsylvania, 231 South 34th Street, Philadelphia, Pennsylvania 19104

E-mail: schelter@sas.upenn.edu

\section{Supporting Information}

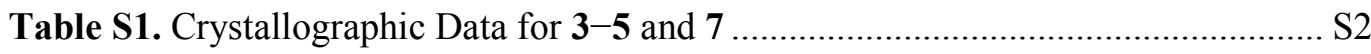

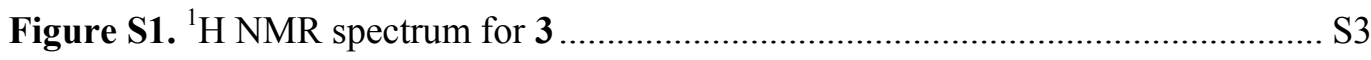

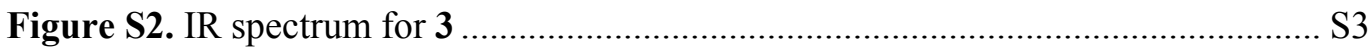

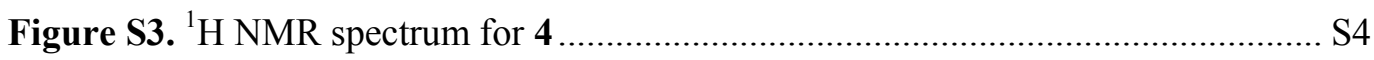

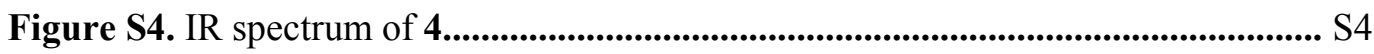

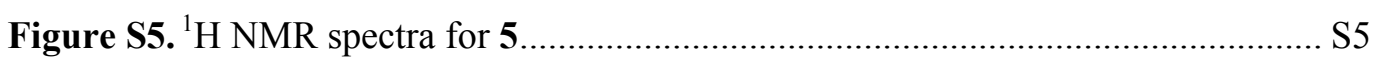

Figure S6. IR spectrum of 5........................................................................................... S5

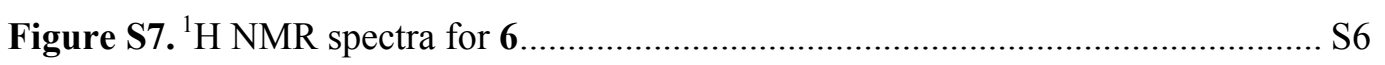

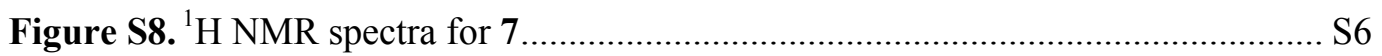


Table S1. Crystallographic Data for 3-5 and 7.

\begin{tabular}{|c|c|c|c|c|}
\hline parameter & 3 & 4 & 5 & 7 \\
\hline formula & $\mathrm{C}_{54} \mathrm{H}_{74} \mathrm{CeN}_{6} \mathrm{NaO}_{4}$ & $\mathrm{C}_{50} \mathrm{H}_{74} \mathrm{CeN}_{6} \mathrm{NaO}_{4}$ & $\mathrm{C}_{57} \mathrm{H}_{78} \mathrm{CeN}_{6} \mathrm{NaO}_{4}$ & $\mathrm{C}_{49} \mathrm{H}_{72} \mathrm{Cs}_{3} \mathrm{O}_{4} \mathrm{Si}_{2}$ \\
\hline Cryst. size [mm] & $0.28 \times 0.10 \times 0.03$ & $0.20 \times 0.05 \times 0.02$ & $0.24 \times 0.08 \times 0.02$ & $0.12 \times 0.12 \times 0.08$ \\
\hline$M \mathrm{r}$ & 1034.30 & 986.26 & 1074.36 & 972.24 \\
\hline$a[\AA]$ & $12.1696(7)$ & $11.9222(11)$ & $13.5231(9)$ & $12.0261(10)$ \\
\hline$b[\AA]$ & $12.1756(8)$ & $19.2992(19)$ & $14.9805(11)$ & $16.8822(13)$ \\
\hline$c[\AA]$ & $19.4986(13)$ & $24.236(2)$ & $15.6696(11)$ & $25.123(2)$ \\
\hline$\alpha[\mathrm{deg}]$ & $96.034(4)$ & $68.518(5)$ & $82.259(4)$ & 90 \\
\hline$\beta$ [deg] & $98.589(3)$ & $86.500(5)$ & $71.974(4)$ & $103.532(4)$ \\
\hline$\gamma[\mathrm{deg}]$ & $108.562(3)$ & $78.672(5)$ & $74.772(4)$ & 90 \\
\hline$V\left[\AA^{3}\right]$ & $2672.0(3)$ & $5087.6(8)$ & $2907.7(4)$ & $4959.0(7)$ \\
\hline $\mathrm{Z}$ & 2 & 4 & 2 & 4 \\
\hline space group & $P-1$ & $P-1$ & $P-1$ & $P 2_{1} / c$ \\
\hline$\rho_{\text {calcd, }}\left[\mathrm{g} \mathrm{cm}^{-3}\right]$ & 1.286 & 1.288 & 1.277 & 1.302 \\
\hline$\mu\left[\mathrm{mm}^{-1}\right]$ & 0.908 & 0.950 & 0.837 & 0.974 \\
\hline $2 \theta$ range $[\mathrm{deg}]$ & $3.58-55.18$ & $3.58-55.54$ & $3.26-55.06$ & $2.94-55.06$ \\
\hline data collected & 87768 & 196856 & 119218 & 120199 \\
\hline no. unique data, $R_{\text {int }}$ & $12069,0.0228$ & $22826,0.1791$ & $13250,0.1064$ & $11407,0.0346$ \\
\hline no. obsd data $[I \geq 2 \sigma(I)]$ & 10755 & 11300 & 8985 & 8796 \\
\hline$R_{1}$ (all data) [a] & 0.0372 & 0.2039 & 0.0388 & 0.0675 \\
\hline$w R_{2}$ (all data) [b] & 0.0606 & 0.3143 & 0.1020 & 0.1177 \\
\hline$S[c]$ & 1.068 & 1.055 & 0.996 & 1.022 \\
\hline no. of variables & 707 & 1152 & 639 & 584 \\
\hline peak/hole $\left[\mathrm{e}^{-3}\right]$ & $1.321 /-1.825$ & $3.624 /-2.297$ & $1.268 /-0.741$ & $2.129 /-1.231$ \\
\hline
\end{tabular}

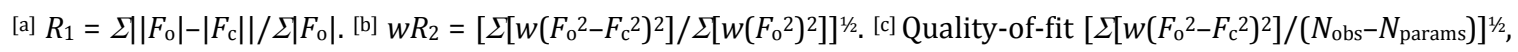
based on all data. 


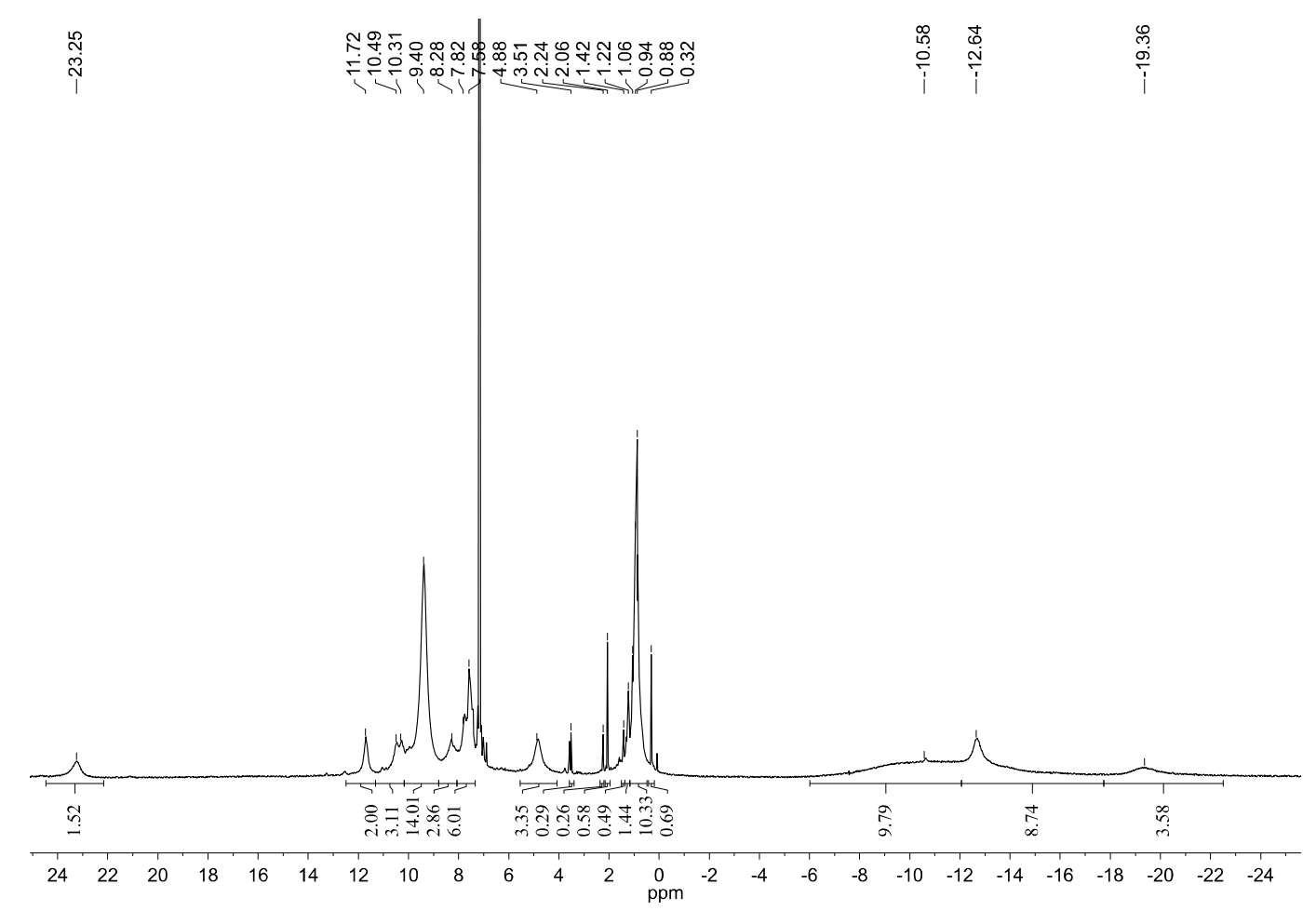

Figure S1. ${ }^{1} \mathrm{H}$ NMR spectrum for 3 in $\mathrm{C}_{6} \mathrm{D}_{6}$.

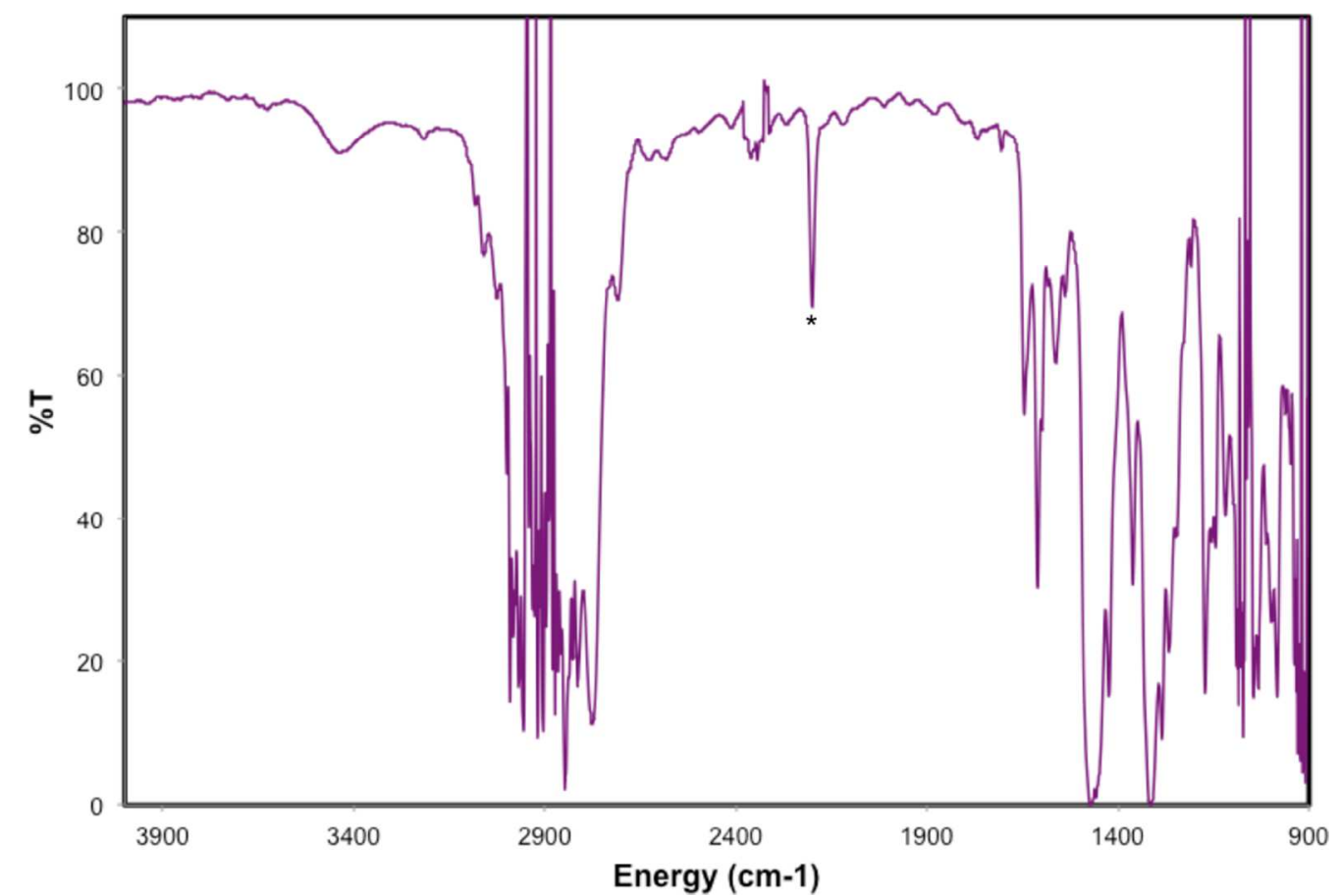

Figure S2. IR spectrum for 3 measured in a THF solution (*- the $\mathrm{C} \equiv \mathrm{C}$ stretch at $2200 \mathrm{~cm}^{-1}$ ). 


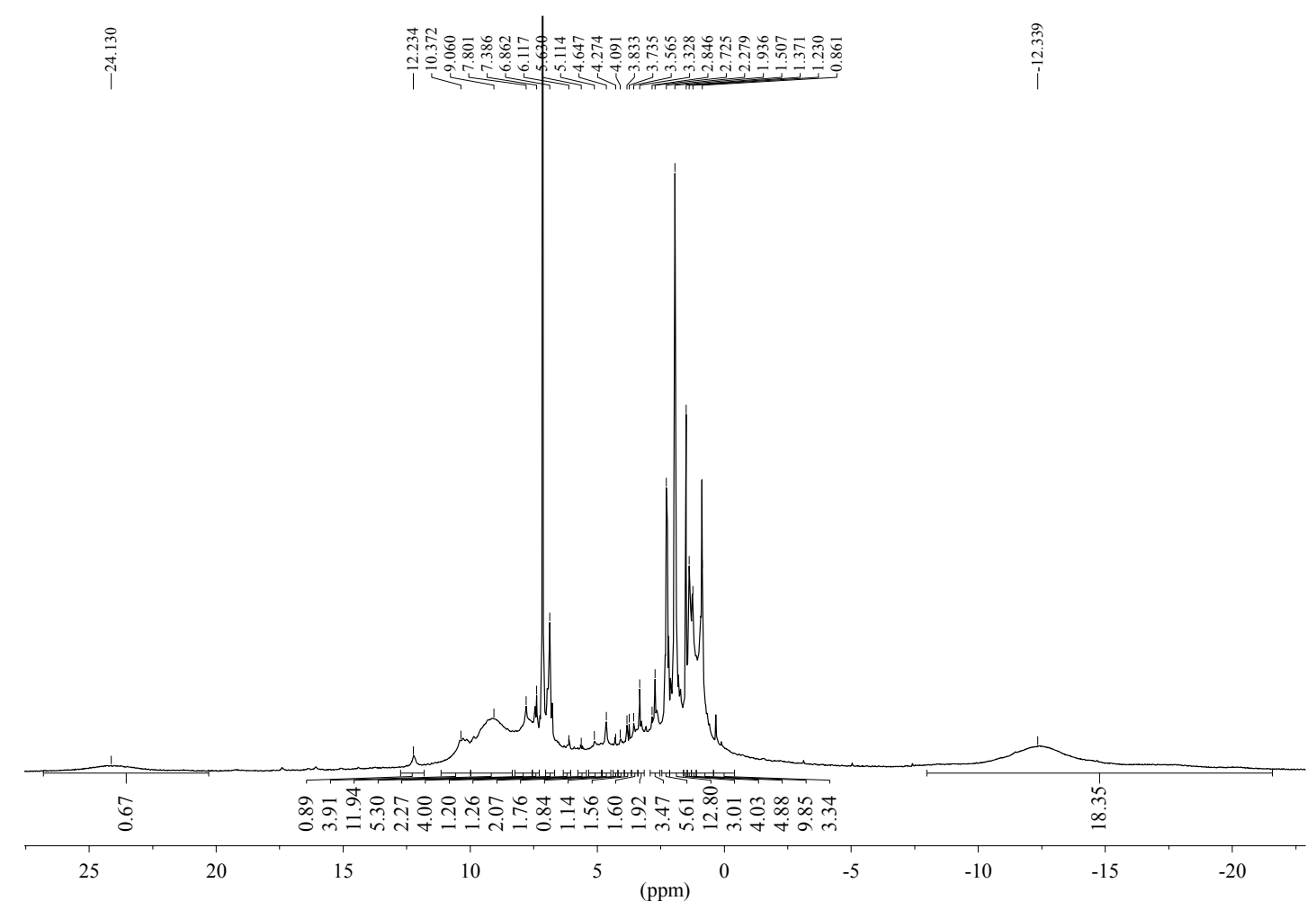

Figure S3. ${ }^{1} \mathrm{H}$ NMR spectrum for 4 in $\mathrm{C}_{6} \mathrm{D}_{6}$.

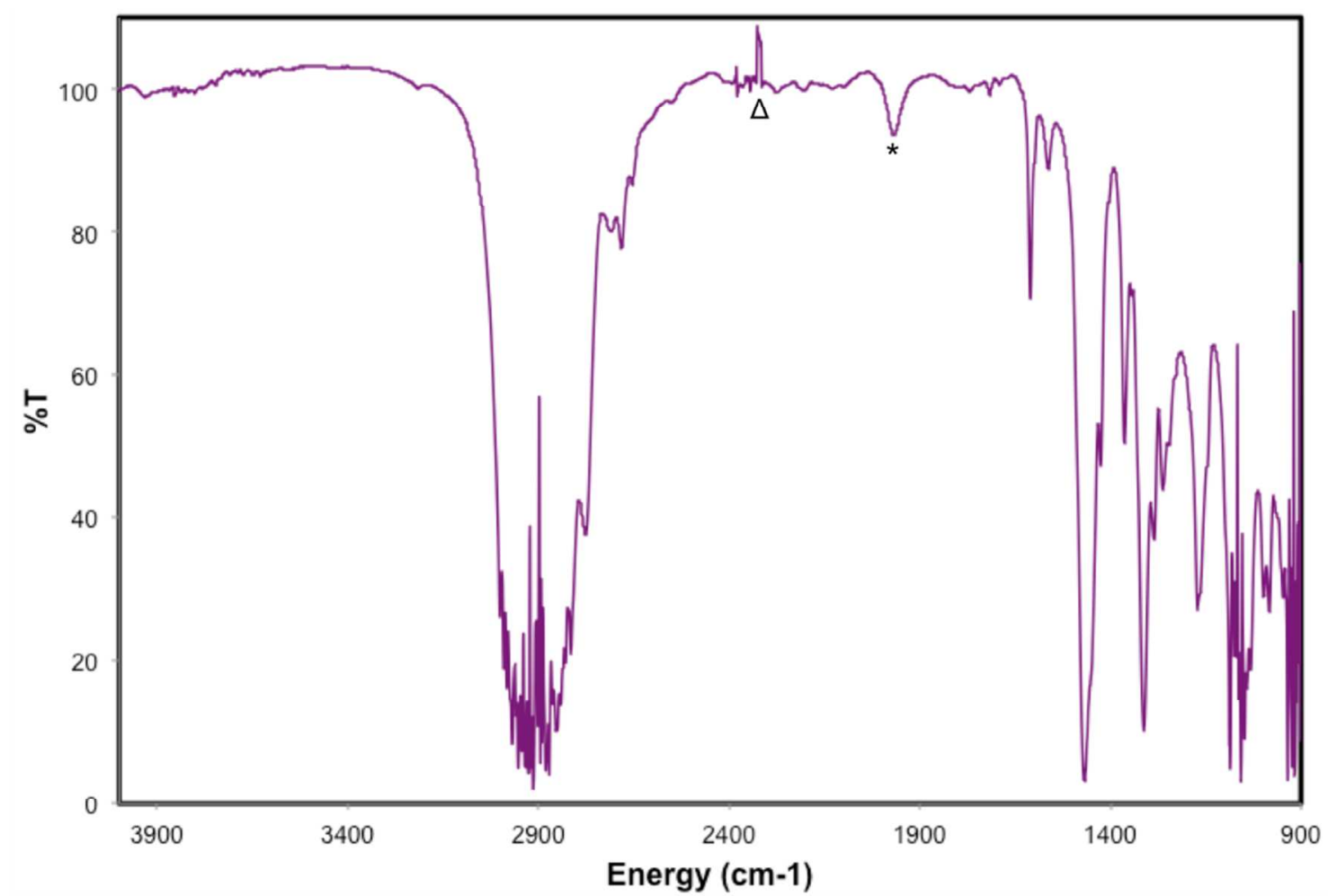

Figure S4. IR spectrum of 4 measured in THF solution. ${ }^{*}$ - the signal of THF solvent. $\Delta-$ experimental artefact. 


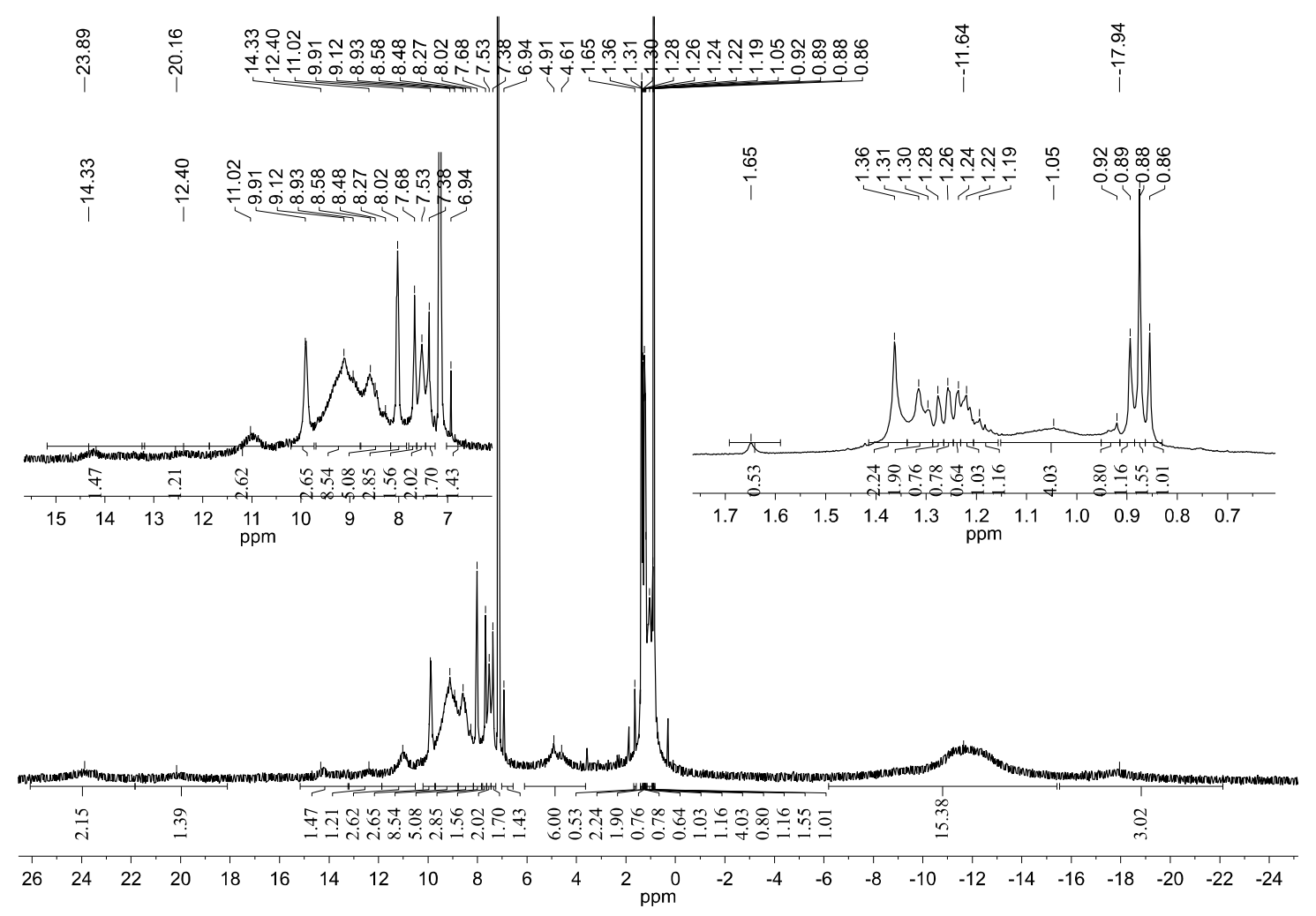

Figure S5. ${ }^{1} \mathrm{H}$ NMR spectrum for 5 in $\mathrm{C}_{6} \mathrm{D}_{6}$.

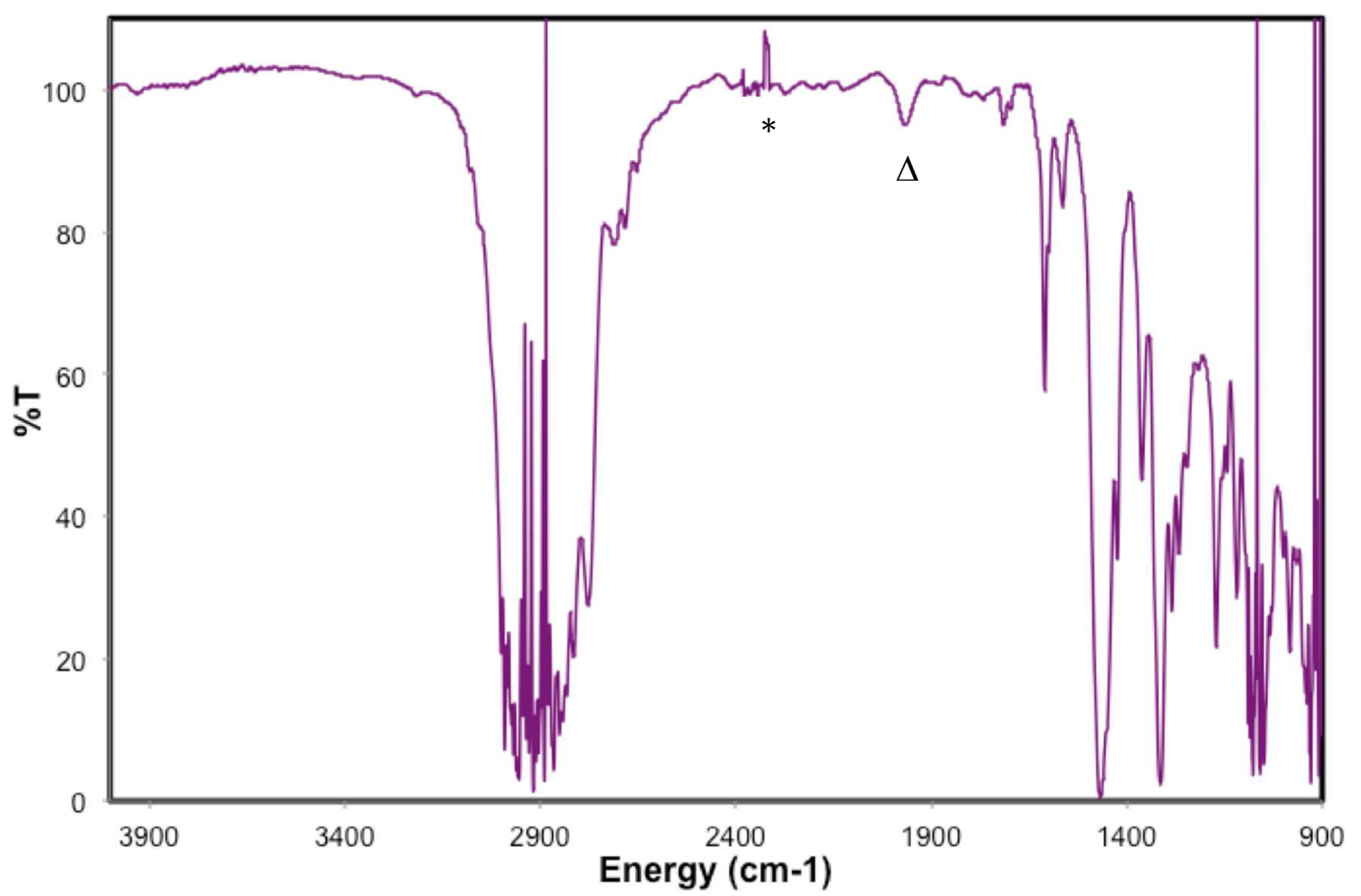

Figure S6. IR spectrum of 5 measured in THF solution. ${ }^{*}$ - the signal of THF solvent. $\Delta-$ experimental artefact. 


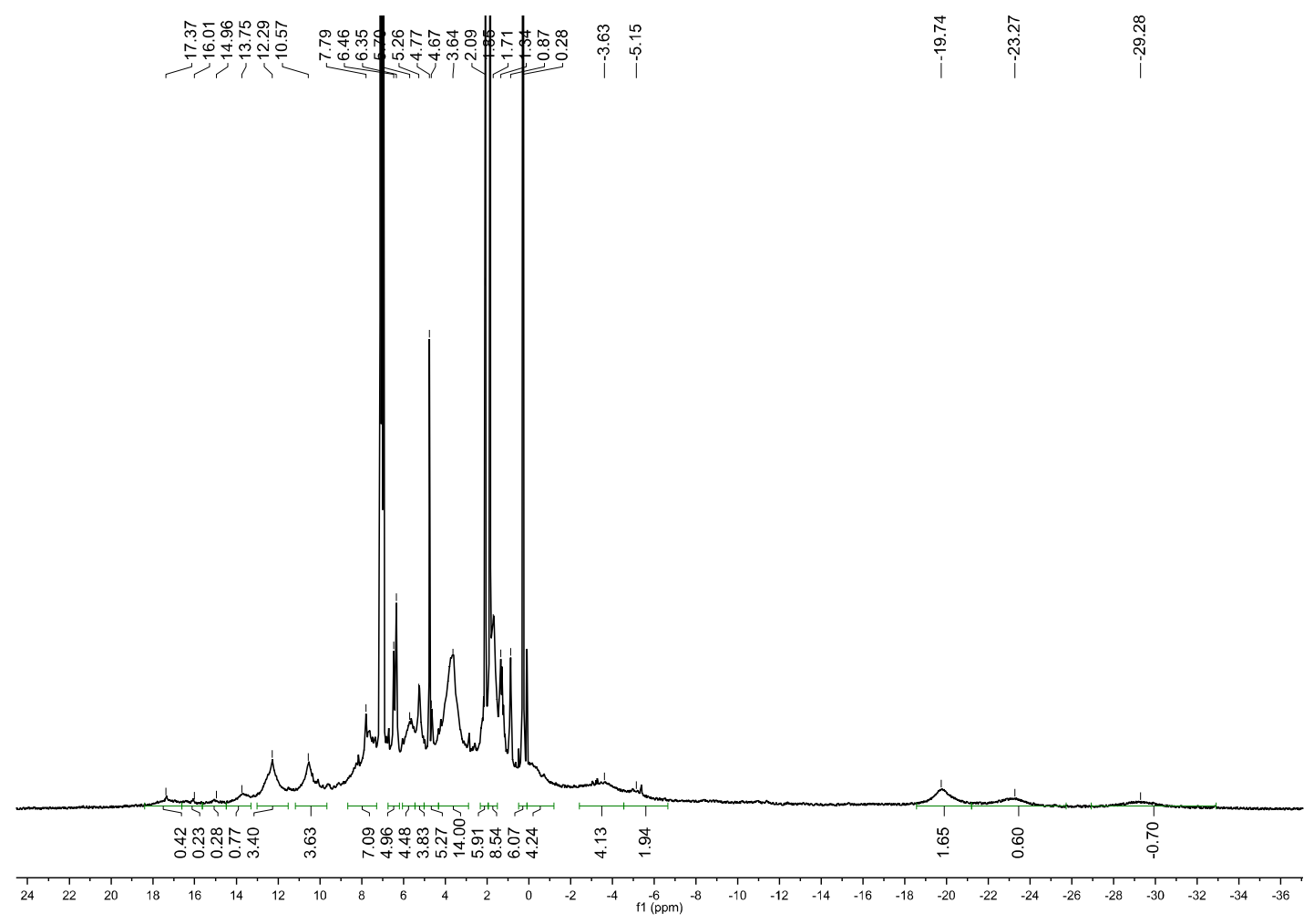

Figure S7. ${ }^{1} \mathrm{H}$ NMR spectrum 6 in toluene- $d_{8}$.

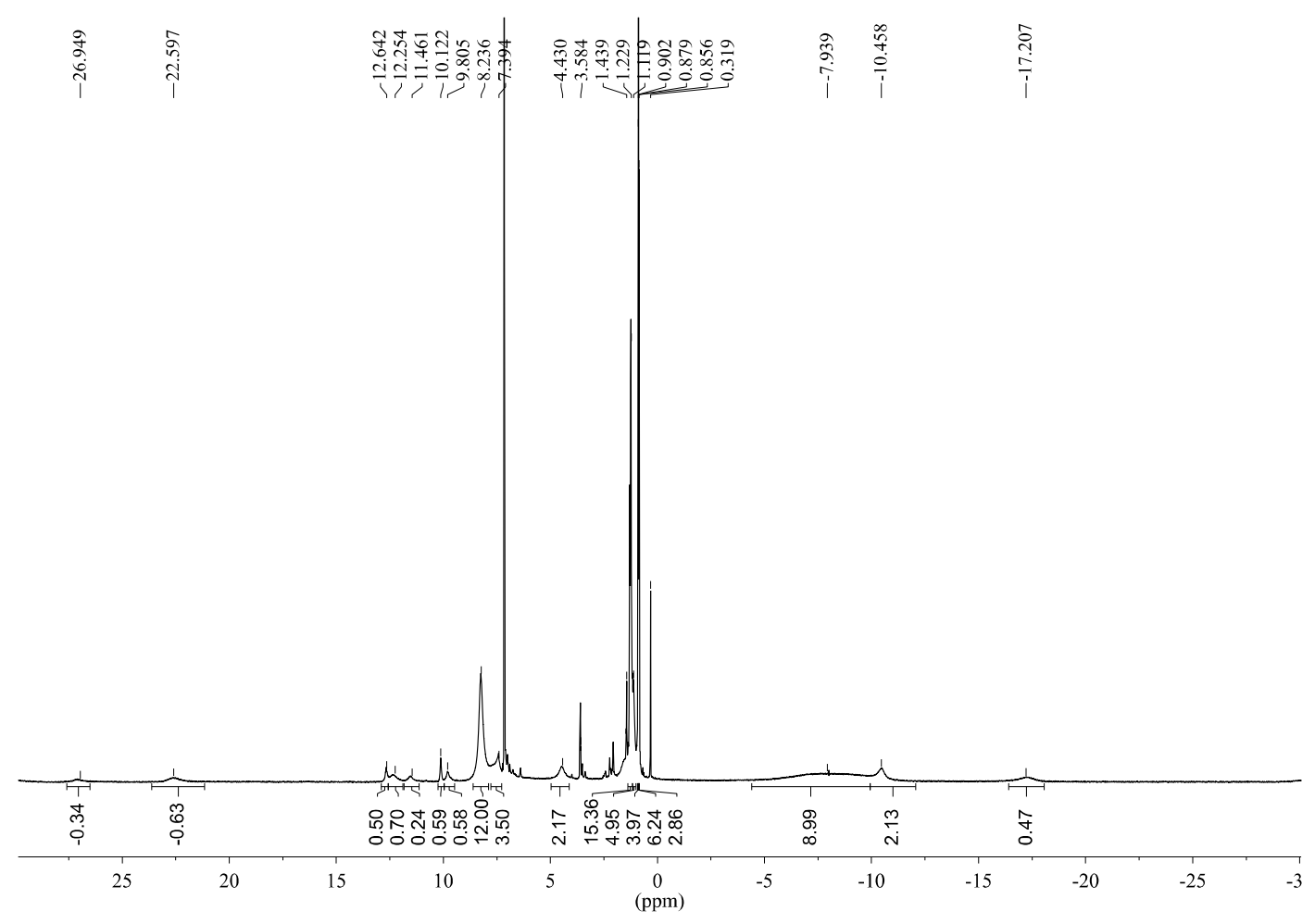

Figure S8. ${ }^{1} \mathrm{H}$ NMR spectrum for 7 in $\mathrm{C}_{6} \mathrm{D}_{6}$. 